Research Paper

\title{
Histone deacetylase inhibitor MGCD0103 causes cell cycle arrest, apoptosis, and autophagy in liver cancer cells
}

\author{
Bo Liao, Quan Sun, Yufeng Yuan, Yuchun Yin, Jianguo Qiao, Ping Jiang ${ }^{\bowtie}$ \\ Department of Hepatopancreatobiliary Surgery, Zhongnan Hospital of Wuhan University, Wuhan 430071, China \\ $\triangle$ Corresponding author: Ping Jiang, Department of Hepatopancreatobiliary Surgery, Zhongnan Hospital of Wuhan University, 169 Donghu Road, Wuhan \\ 430071, China. Phone/Fax: +86-027-67812959; E-mail address: jiangp2003@whu.edu.cn \\ (C) The author(s). This is an open access article distributed under the terms of the Creative Commons Attribution License (https://creativecommons.org/licenses/by/4.0/). \\ See http://ivyspring.com/terms for full terms and conditions.
}

Received: 2019.10.14; Accepted: 2019.12.01; Published: 2020.01.29

\begin{abstract}
Background: Liver cancer is a common cause of cancer-related death all over the world. MGCD0103, a histone deacetylase inhibitor, exerts antitumor effect on various cancers. However, its role in liver cancer remains unclear.

Methods: The effect of MGCD0103 on HepG2 and Huh7 cells was verified by several experiments such as cell viability assay, colony formation assay, cell cycle analysis, apoptosis analysis, reactive oxygen species (ROS) assay, western blotting, immunohistochemistry, and xenograft assay.

Results: Cell viability and colony formation assays showed that MGCD0103 inhibited the proliferation of liver cancer cells in vitro. Flow cytometry and western blotting analysis demonstrated that MGCD0103 induced G2/M phase arrest and mitochondrial-related apoptosis. A pan-caspase inhibitor and ROS scavenger inhibited apoptosis induced by MGCD0103. What's more, MGCD0103 led to autophagy associated with cell death and an autophagy inhibitor inhibited apoptosis and autophagy induced by MGCD0103. Ultimately, MGCD0103 attenuated tumor growth but did not show significant systemic toxicity in animal model.

Conclusions: MGCD0103 suppressed the growth of liver cancer cells in vitro and in vivo. It could serve as a novel therapeutic approach for liver cancer.
\end{abstract}

Key words: liver cancer, MGCD0103, cell growth, cell cycle arrest, apoptosis, autophagy

\section{Introduction}

Liver cancer is one of the leading causes of cancer-related deaths worldwide and China alone accounts for about half of the total number of cases and deaths [1]. Liver cancer at early stages might be curable by liver transplantation or surgical resection [2]. However, there are still no curative treatments for advanced liver cancer due to its resistance to chemotherapy. Sorafenib have shown survival benefits for patients with advanced liver cancer [3]. However, it is not widely used in Asia due to the expensive cost and increased adverse events [4, 5], and therefore, novel therapeutic drugs need to be developed for advanced liver cancer.

Genome instability and mutation are one of the hallmarks of cancer [6]. Inactivation of tumor suppressor genes can be acquired through epigenetic mechanisms such as histone modifications [7]. Histone acetyl transferase (HAT) and histone deacetylase (HDAC) are the two enzymes controlling the level of acetylation of histones [8]. HDAC removes acetyl groups from histone, causes gene silencing and exerts a pro-oncogenic effect. The balance between HAT and HDAC activity is disrupted in many cancers. HDAC inhibitors repress the activity of HDAC enzymes and therefore exert anticancer activity. HDAC inhibitors promote acetylation of histones and non-histone protein substrates. The HDAC enzymes are the targets of the HDAC inhibitors and grouped into four types. Class I HDAC enzymes include HDAC1, HDAC2, HDAC3, and 
HDAC8. Class II HDACs include HDAC4, HDAC5, HDAC6, HDAC7, HDAC9, and HDAC10. Class III HDACs, also named sirtuins, include seven human isoenzymes (SIRT 1-7). Class IV HDAC is HDAC11.

Several HDAC inhibitors such as vorinostat, romidepsin, belinostat, and panobinostat have been approved by the FDA for the treatment of cancer [9]. After that, more and more HDAC inhibitors are in different phases of clinical trials and MGCD0103 (mocetinostat) is one of them. MGCD0103, shown in Fig. 1A, was developed in Canada by MethylGene [10]. It is a synthesized HDAC inhibitor, highly specific for classes I and IV HDACs. This compound selectively inhibits HDAC1, HDAC2, HDAC3 and HDAC11. It was studied in Phase I/ II trials for solid tumors and hematologic malignancies [11-13]. Preclinical studies have demonstrated that it can induce apoptosis and cell cycle blockade in various kinds of cancer [10]. Other studies have shown that it can induce or suppress autophagy in different cancers
$[14,15]$. However, its role in the treatment of liver cancer remains still unclear.

In this study, we explored the effect of MGCD0103 on growth, apoptosis, cell cycle, and autophagy in liver cancer cells in vitro and in vivo. The related molecular mechanisms were also investigated.

\section{Materials and Methods}

\section{Cell lines and cell cultures}

HepG2 (a hepatoma cell line) and Huh7 (a hepatocellular carcinoma cell line) cell lines were purchased from China Center for Type Culture Collection (CCTCC, Wuhan, China). Cell lines were maintained in high-glucose Dulbecco's modified Eagle's medium (DMEM) (Gibco, NY, USA) containing 10\% fetal bovine serum (FBS) (Gibco, NY, USA). Cells were cultured at cell culture incubator with $37^{\circ} \mathrm{C}$ and $5 \% \mathrm{CO} 2$.
A

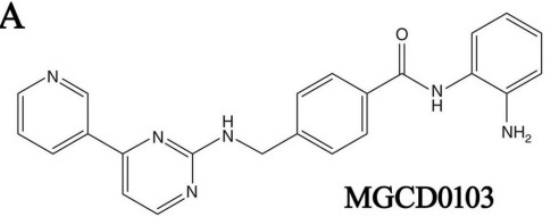

C

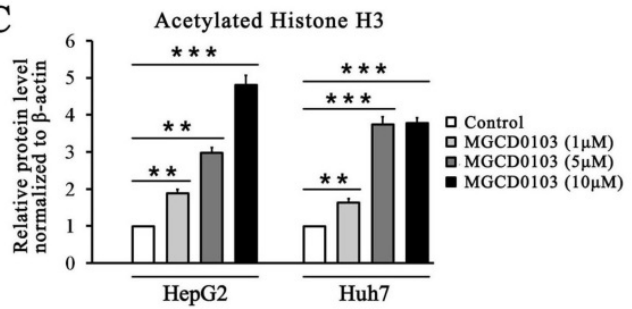

D
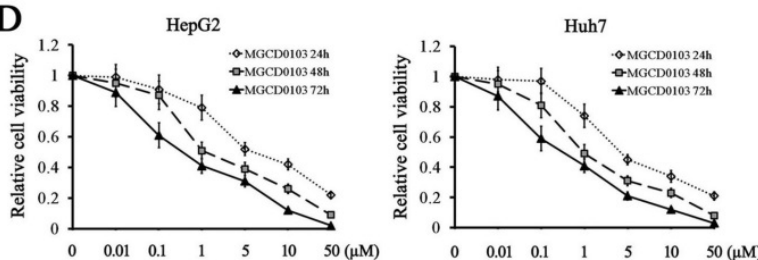

B
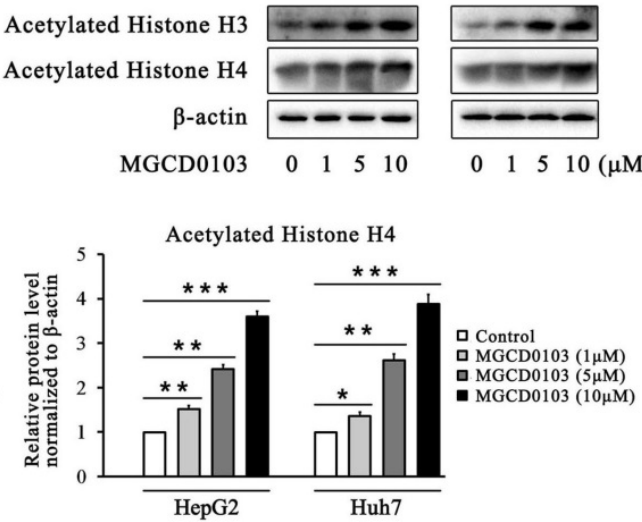

$\mathrm{E}$

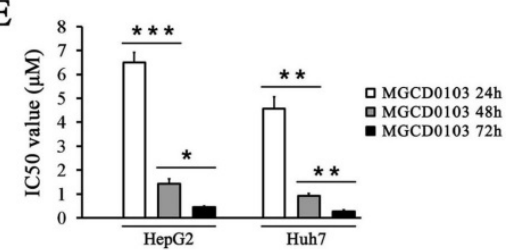

F

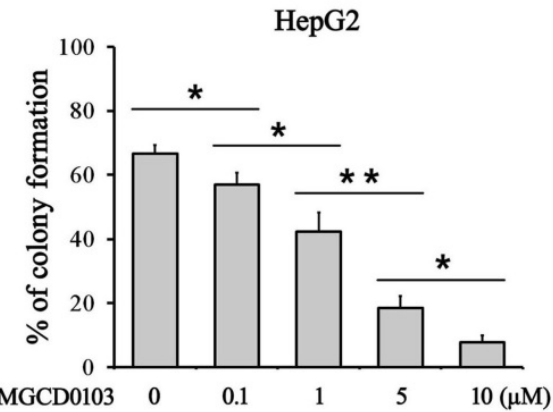

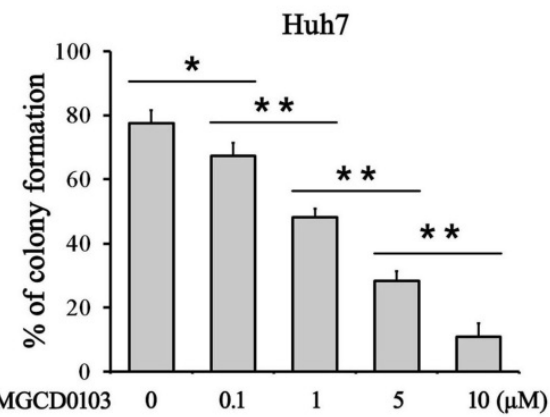

Figure 1. MGCDO103 upregulates acetylated histone $\mathrm{H} 3 / 4$ and inhibits the growth of liver cancer cells in a dose-dependent manner. (A) The chemical structure of MGCD0103. (B, C) HepG2 and Huh7 cells were treated with increasing concentrations of MGCD0103 for $48 \mathrm{~h}$, and the expression of acetylated histone $\mathrm{H} 3$ and $\mathrm{H} 4$ was detected by western blotting. (D) HepG2 and Huh7 cells were treated with increasing concentrations $(0.01,0.1,1,5,10$, and $50 \mu \mathrm{M})$ of MGCD0103 for $24 \mathrm{~h}, 48 \mathrm{~h}$, or $72 \mathrm{~h}$. Cell viability was assessed using the CCK-8 assay. (E) The IC50 values of MGCD0103 in HepG2 and Huh7 cell lines were calculated. (F) Colony formation assay. HepG2

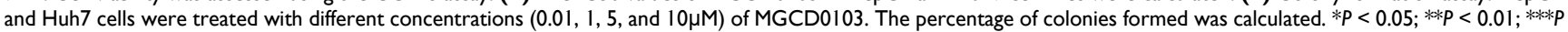
$<0.001$ 


\section{Drugs and reagents}

MGCD0103, Z-VAD-FMK, and 3-Methyladenine (3-MA) were purchased from Selleck Chemicals (TX, USA). Crystal violet, 5-FU, N-Acetyl Cysteine (NAC), and DMSO were purchased from Sigma-Aldrich (MO, USA). Cell Counting Kit-8 (CCK-8) was purchased from Dojindo (Kumamoto, Japan).

\section{Cell viability assay}

HepG2 $\left(1 \times 10^{3}\right.$ cells/well $)$ and Huh7 $\left(1.5 \times 10^{3}\right.$ cells/well) cells were plated in 96-well plates and treated with increasing concentrations $(0.01,0.1,1,5$, 10 , and $50 \mu \mathrm{M}$ ) of MGCD0103 for different lengths of time ( $24 \mathrm{~h}, 48 \mathrm{~h}$, and $72 \mathrm{~h})$ to generate cell viability curves. CCK- 8 reagent was added for $2 \mathrm{~h}$ and the optical density (OD) values were measured at $450 \mathrm{~nm}$. The half maximal inhibitory concentration (IC50) was calculated by SPSS software.

\section{Colony formation assay}

Colony formation assay was carried out as described previously [16]. HepG2 (500 cells/well) and Huh7 (500 cells/well) cells were plated in 6-well plates and treated with different concentrations (0.1, 1, 5, and $10 \mu \mathrm{M}$ ) of MGCD0103 for $48 \mathrm{~h}$. Media were refreshed every other day. The plates were stained with crystal violet and the images were acquired on day 14. The numbers of colonies were counted and analyzed by Alpha Innotech Imaging system (Alphatron Asia Pte Ltd, Singapore).

\section{Western blotting}

Western blotting was performed as described previously [17]. The primary antibodies against acetylated histone $\mathrm{H} 3$, acetylated histone $\mathrm{H} 4$, and $\beta$-actin were purchased from Santa Cruz Biotechnology (CA, USA). The primary antibodies against $\mathrm{p} 21$, p27, cdc25C, cyclin B1, Bim, Bax, Bcl-2, Bcl-xL, RAGE, and LC3 were purchased from Epitomics (CA, USA). The primary antibodies against p-cdc25C, cdc2, p-cdc2, Cyto-C, cleaved caspase-9, cleaved caspase-3, cleaved caspase-7, cleaved-PARP, PARP, PI3KC3, Beclin 1, and p62 were purchased from Cell Signaling Technology (MA, USA). All secondary antibodies were purchased from Jackson Immuno Research Laboratories (PA, USA).

\section{Cell cycle and apoptosis analysis}

The flow cytometry analysis was carried out as described previously [18]. For cell cycle analysis, HepG2 and Huh7 cells were treated with MGCD0103 for $48 \mathrm{~h}$. A total of $1 \times 10^{6}$ cells were analyzed by FACSAria Cell Cytometer (BD Biosciences, CA, USA). For apoptosis analysis, $1 \times 10^{5}$ cells were analyzed. All data were analyzed by CellQuest software (BD
Biosciences, CA, USA).

\section{ROS assay}

The ROS assay was performed as described previously [19]. HepG2 and Huh7 cells were treated with MGCD0103 in the presence or absence of NAC (5 $\mathrm{mM}$ ) for $48 \mathrm{~h}$. The fluorescent probe DCFH-DA was used to evaluate intracellular ROS levels. Cells were analyzed by FACSAria Cell Cytometer.

\section{Animal model}

Animal experiments were performed as previously described [16, 20]. All procedures in animal experiments were approved by the Committee on the Ethics of Animal Experiments of Zhongnan Hospital, Wuhan University. HepG2 cells were subcutaneously injected into the mice. MGCD0103 treatment started on day 9 after injection when the tumors were palpable. MGCD0103 was dissolved in DMSO and dosed per os (p.o.) daily. All the animals were sacrificed by cervical dislocation on day 24 .

Subcutaneous tumors were dissected, removed and conserved for proliferation and apoptosis analysis. AST, ALT, Cr, and BUN levels in mice blood were measured by Reflotron Plus system (Roche, IN, USA). Organ tissues including heart, liver, spleen, lung, and kidney were removed and sectioned.

\section{Immunohistochemistry analysis}

Immunohistochemistry was performed as previously described [16, 20]. Ki-67 primary antibody was purchased from Dako (Golstrup, Denmark). Tumor tissues were incubated with primary antibodies against Ki-67 and cleaved caspase-3, then incubated with a secondary antibody. Organ tissues were stained with H\&E for the histological analysis.

\section{Statistical analyses}

Statistical analyses were performed by SPSS 13.0 ( IL, USA). All experiments were carried out at least three independent times. The results were expressed as the mean $\pm \mathrm{SD}$. Comparisons between the different groups were analyzed by one-way ANOVA, and $P<$ 0.05 was considered to be statistically significant.

\section{Results}

\section{MGCD0103 increases the acetylation of histone $\mathrm{H} 3$ and $\mathrm{H} 4$ in liver cancer cell lines}

The effect of MGCD0103 on the acetylation of histone $\mathrm{H} 3$ and histone $\mathrm{H} 4$ was evaluated in HepG2 and Huh7 cells. The western blotting results showed that treatment with increasing concentrations of MGCD0103 for $48 \mathrm{~h}$ increased the acetylation level of histone $\mathrm{H} 3$ and histone $\mathrm{H} 4$ in HepG2 and Huh7 cell lines in a dose-dependent manner (Fig. 1B and C). 
A
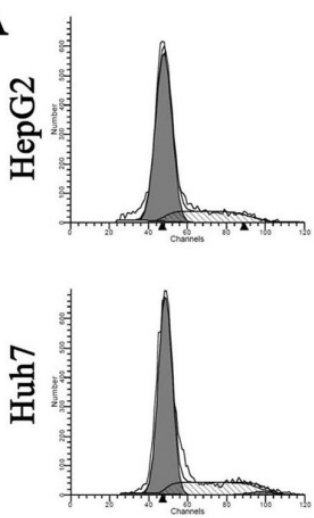

$5-\mathrm{FU}(10 \mu \mathrm{M})$
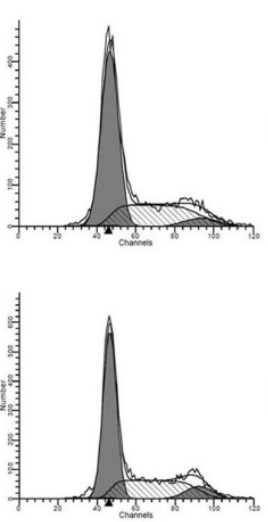

Control
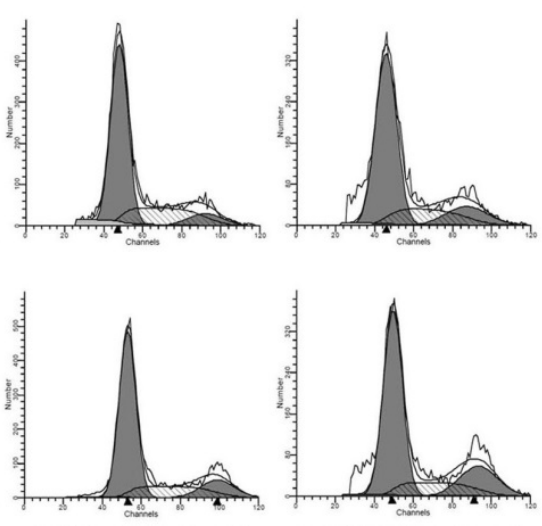

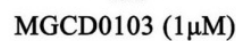

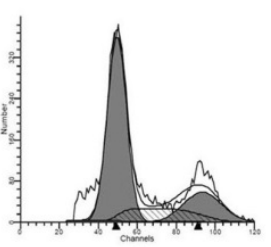

$\operatorname{MGCD} 0103(5 \mu \mathrm{M})$
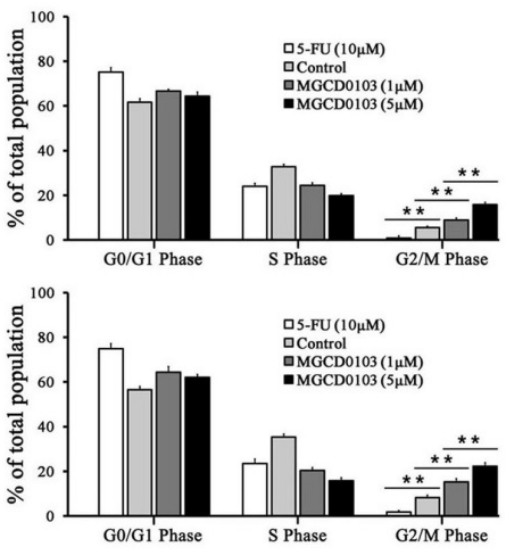

B

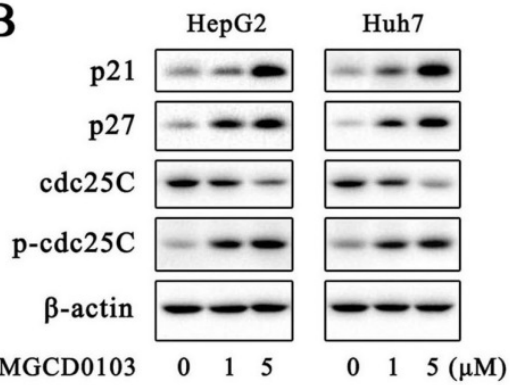

C

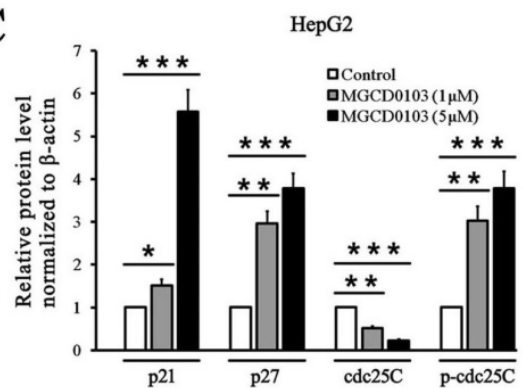

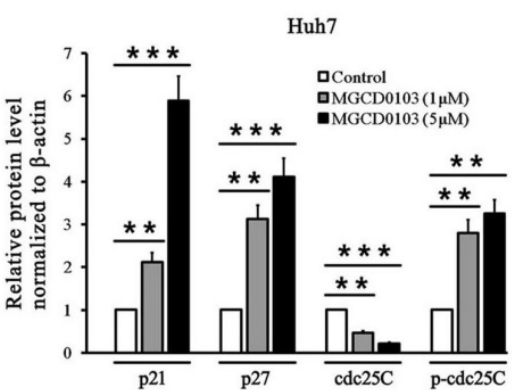

D

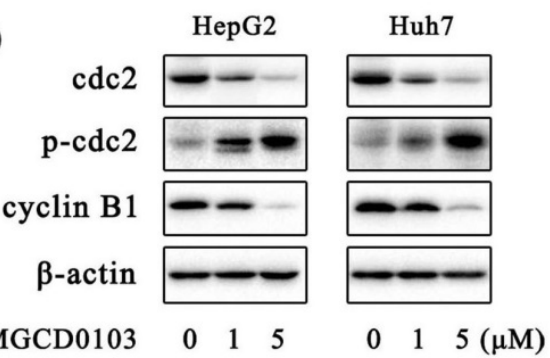

$\mathrm{E}$

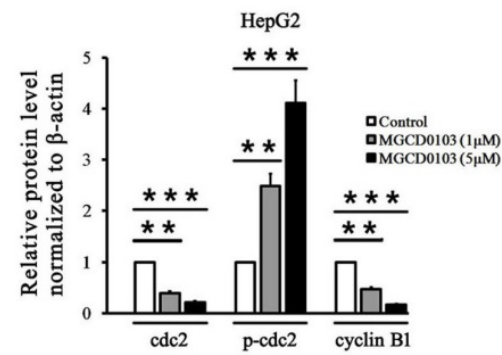

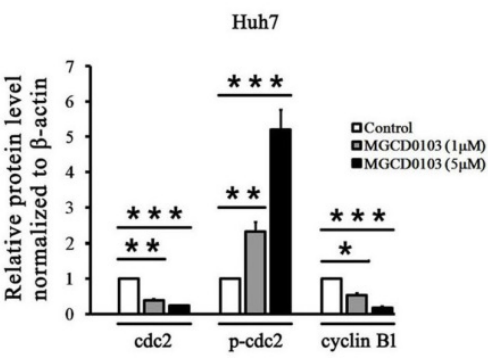

Figure 2. MGCD0103 causes G2/M phase arrest in liver cancer cells. (A) HepG2 and Huh7 cells were treated with 5 -FU (10 $\mu$ M) and MGCD0103 ( $\mu$ M and $5 \mu M)$ for 48h. Cell cycle distribution was then assessed using flow cytometry. (B-E) Western blotting analysis of p21, p27, cdc25C, p-cdc25C, cdc2, p-cdc2, and cyclin B1 after MGCD0103 treatment. $* P<0.05 ; * * P<0.01 ; * * * P<0.001$

\section{MGCD0103 suppresses the growth of liver cancer cells}

To investigate the inhibitory effect of MGCD0103 on liver cancer cells, HepG2 and Huh7 cell lines were treated with MGCD0103. The CCK-8 assay demonstrated that MGCD0103 exhibited dosedependent and time-dependent cytotoxic effects on HepG2 and Huh7 cells (Fig. 1D and E). The IC50 values of MGCD0103 in HepG2 cells for different lengths of time $(24 \mathrm{~h}, 48 \mathrm{~h}$, and $72 \mathrm{~h})$ were $6.497 \pm 0.431$ $\mu \mathrm{mol} / \mathrm{L}(\mu \mathrm{M}), 1.427 \pm 0.206 \mu \mathrm{M}$, and $0.453 \pm 0.055 \mu \mathrm{M}$, respectively, and those in Huh7 cells were $4.567 \pm$ $0.496,0.920 \pm 0.096$, and $0.277 \pm 0.061 \mu \mathrm{M}$, respectively (Fig. 1E). The results indicated that MGCD0103 exerted anti-proliferative activity against liver cancer cells. Colony formation assay showed that MGCD0103 reduced the colony numbers of HepG2 and Huh7 cells in a dose-dependent manner (Fig. 1F).
The colony formation rates of HepG2 cells treated with increasing concentrations $(0,0.1,1,5$, and $10 \mu \mathrm{M})$ of MGCD0103 were $66.54 \pm 2.71 \%, 56.91 \pm 3.68 \%, 42.37$ $\pm 5.93 \%, 18.41 \pm 3.76 \%$, and $7.72 \pm 2.15 \%$, respectively, and those in Huh7 cells were $77.50 \pm 4.03,67.22 \pm 4.02$, $48.25 \pm 2.65,28.38 \pm 3.01$, and $10.86 \pm 4.20 \%$, respectively (Fig. 1F).

\section{MGCD0103 induces cell cycle arrest in liver cancer cells}

5-FU, as the positive control, caused cell cycle arrest in HepG2 and Huh7 cells at G0/G1 phase (Fig. $2 \mathrm{~A})$. The proportion of cells at G2/M phase was decreased after treatment with 5-FU (Fig. 2A). Compared with the control group, MGCD0103 caused G2/M cell cycle arrest in HepG2 and Huh7 cells (Fig. 2A). The proportions at G2/M phase of HepG2 cells treated with increasing concentrations $(0,1$, and $5 \mu \mathrm{M})$ of MGCD0103 were $5.55 \pm 0.58 \%, 8.90 \pm 0.90 \%$, and 
$15.72 \pm 1.14 \%$, respectively, and those of Huh7 cells were $8.16 \pm 1.18,15.26 \pm 1.45$, and $22.20 \pm 1.72 \%$, respectively (Fig. 2A). Some related proteins were tested by western blotting. MGCD0103 upregulated the protein levels of p21, p27, p-cdc25C, and p-cdc2, while downregulated those of cdc25C, cdc2, and cyclin B1 in a dose-dependent manner (Fig. 2b-e).

\section{MGCD0103 triggers apoptosis in liver cancer cells}

The flow cytometry analysis showed that the apoptotic rates of HepG2 and Huh7 cells were elevated after treatment with MGCD0103 in a dose-dependent manner (Fig. 3a). The apoptotic rates of HepG2 cells treated with increasing concentrations $(0,1$, and $5 \mu \mathrm{M})$ of MGCD0103 were $7.84 \pm 1.03 \%$, $13.63 \pm 2.03 \%$, and $23.47 \pm 1.69 \%$, respectively, and those of Huh7 cells were $6.45 \pm 0.41,18.78 \pm 1.27$, and $29.48 \pm 2.13 \%$, respectively (Fig. 3 A). Several apoptosis- related proteins were detected by western blotting. MGCD0103 downregulated the expressions of Bcl-2 as well as Bcl-xL, and upregulated those of Bim, Bax, Cyto-C, cleaved caspase-9, cleaved caspase-3, cleaved caspase-7, and cleaved-PARP in a dose-dependent manner (Fig. 3B-E). The above alterations indicated the activation of the mitochondria apoptosis pathway.

To further evaluate the effect of MGCD0103 on the intrinsic apoptotic pathway, HepG2 and Huh7 cells were pretreated with the caspase inhibitor Z-VAD-FMK $(20 \mu \mathrm{M})$ before treatment with MGCD0103. The pretreatment of Z-VAD-FMK decreased the apoptotic rate caused by MGCD0103 from $28.47 \pm 2.85$ to $17.74 \pm 1.32 \%$ in HepG2 cells and from $33.29 \pm 2.93$ to $20.06 \pm 2.02 \%$ in Huh7 cells (Fig. 4A). Western blotting showed that Z-VAD-FMK attenuated the cleavage of PARP induced by MGCD0103 (Fig. 4B and C).
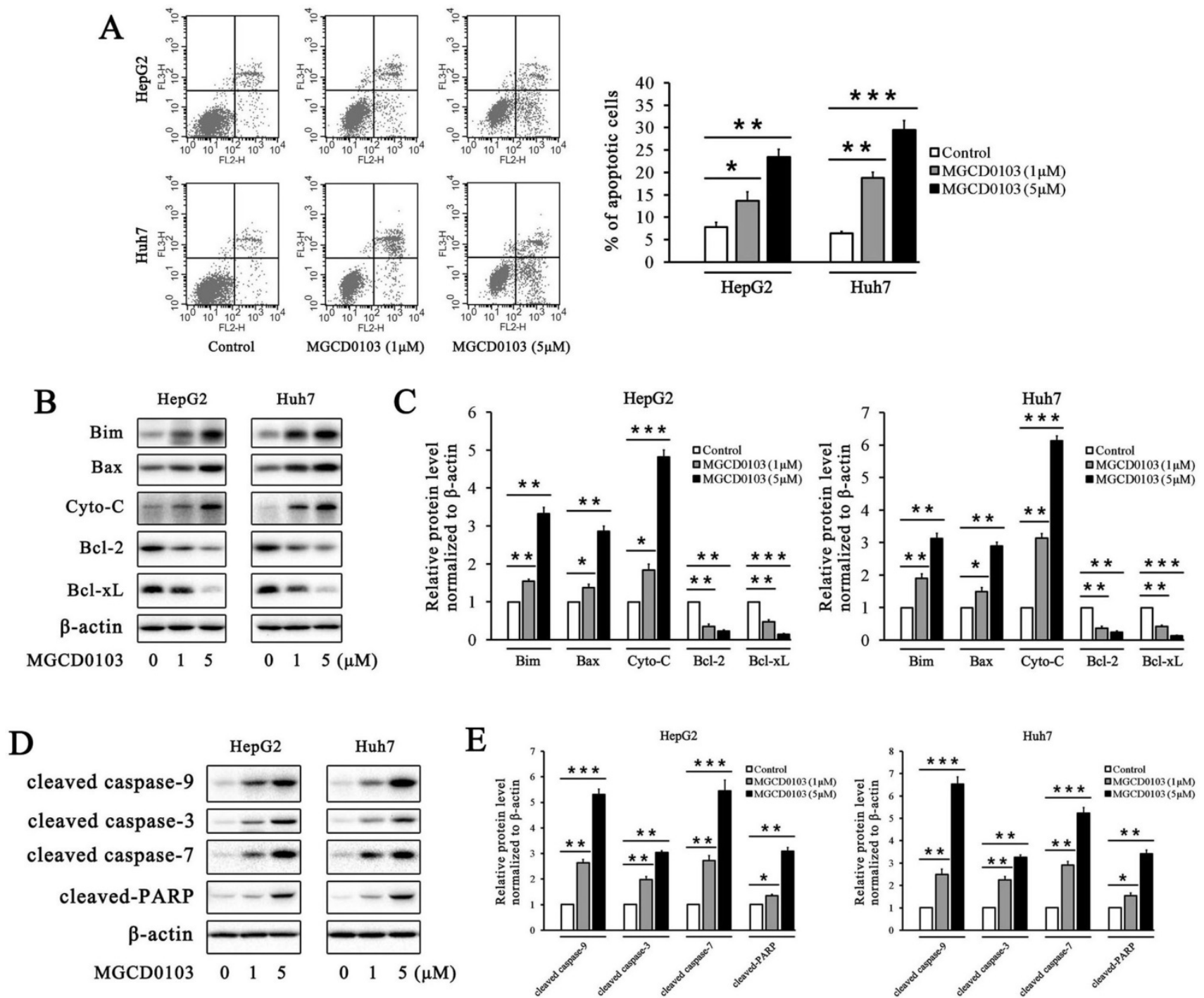

Figure 3. MGCD0103 causes apoptosis in liver cancer cells. (A) HepG2 and uh7 cells were treated with MGCD0103 (1 $\mu$ M and $5 \mu M$ ) for 48 h. Apoptosis was evaluated by flow cytometry. Apoptotic rate was then calculated. (B-E) Western blotting analysis of Bim, Bax, Cyto-C in cytosol, Bcl-2, Bcl-xL, cleaved caspase-9, cleaved caspase-3, cleaved caspase-7, and cleaved-PARP after MGCD0103 treatment. $* P<0.05 ; * * p<0.01$; ***p<0.001 
A
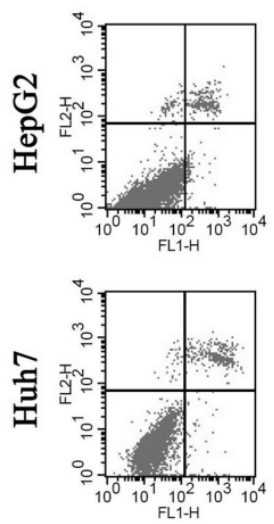

MGCD0103

Z-VAD-FMK -

B
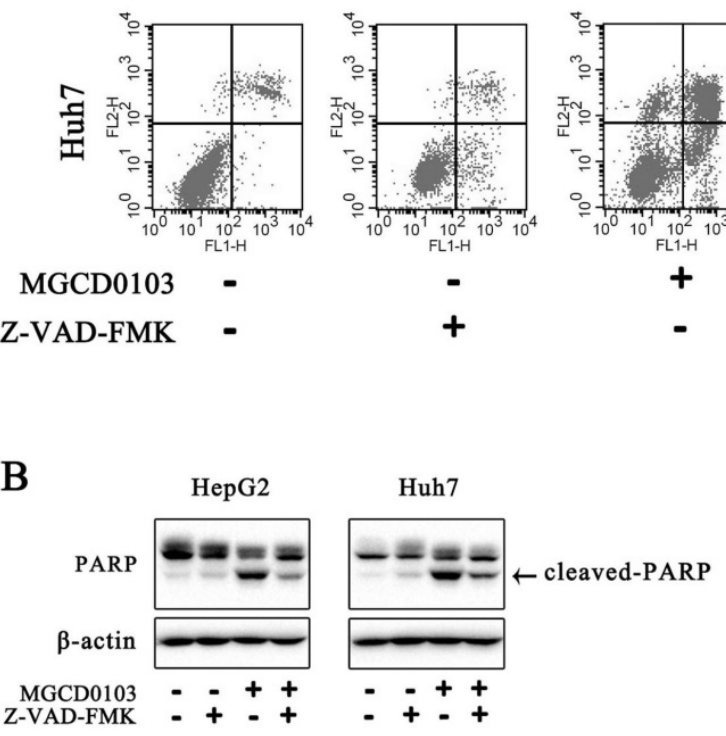
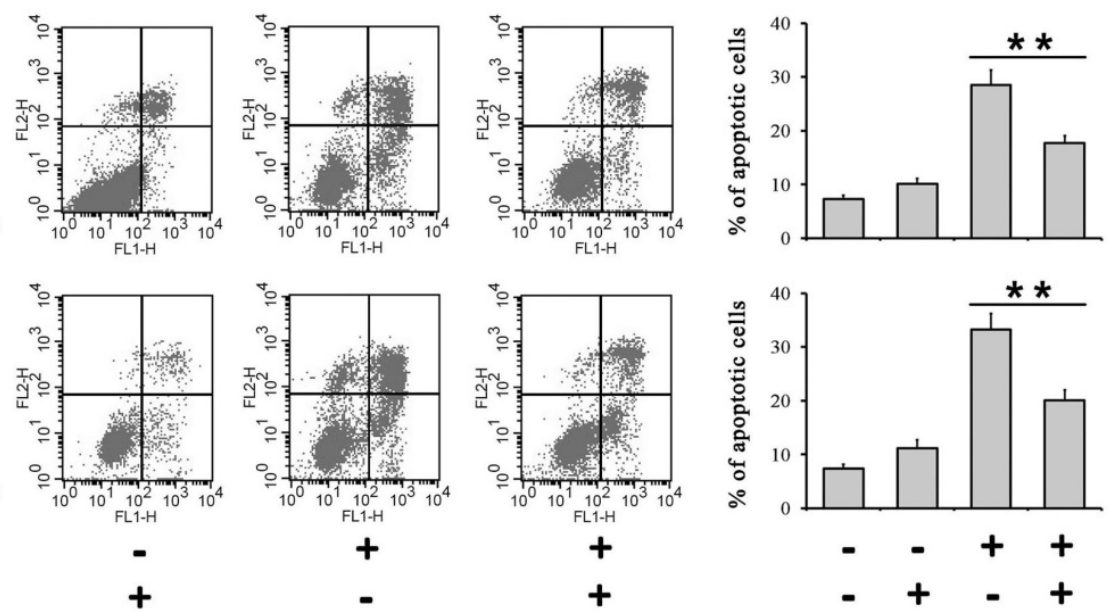

C

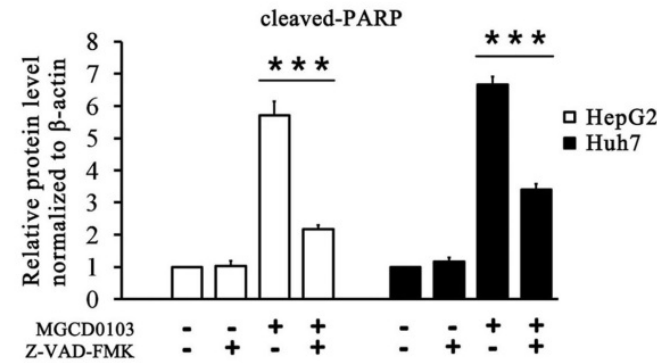

Figure 4. Caspase inhibitor Z-VAD-FMK partially inhibited apoptosis induced by MGCD0103 in liver cancer cells. (A) HepG2 and HUH7 cells were treated with $20 \mu \mathrm{M}$ Z-VAD-FMK for $1 \mathrm{~h}$ and then $5 \mu \mathrm{M}$ MGCD0103 for the next $48 \mathrm{~h}$. Apoptosis was evaluated by flow cytometry. (B, C) Western blotting analysis of cleaved-PARP after MGCD0103 and/or Z-VAD-FMK treatment. **P $<0.01$; ***P $<0.001$

\section{ROS participates in the apoptosis induced by MGCD0103 in liver cancer cells}

ROS produced by the mitochondria participates in the regulation of apoptosis, and inhibition of apoptosis by antiapoptotic Bcl-2 is related to the protection against ROS [21]. The ROS assay demonstrated that ROS levels in HepG2 and Huh7 cells were dose-dependently increased after treatment with MGCD0103 (Fig. 5A). Cells were then pre-treated with NAC ( $5 \mathrm{mM})$ before treatment with MGCD0103 for a further $48 \mathrm{~h}$. Pretreatment with NAC led to significantly decreased MGCD0103-induced ROS levels (Fig. 5B). HepG2 and Huh7 cells were pretreated with NAC $(5 \mathrm{mM})$ before treatment with MGCD0103 to verify if ROS may play a role in apoptosis or G2/M phase arrest induced by MGCD0103. The apoptotic effect was reversed by the addition of NAC which decreased the apoptotic rate from $28.09 \pm 1.84 \%$ caused by MGCD0103 to $19.45 \pm$ $1.74 \%$ in HepG2 cells and from $33.94 \pm 2.43$ to $22.19 \pm$ $1.98 \%$ in Huh7 cells (Fig. 6A). However, NAC did not influence G2/M phase arrest caused by MGCD0103 (Fig. 6B). Western blotting showed that NAC restored the expression of Bcl-2 reduced by MGCD0103, but failed to impact on the decreased expression of cyclin B1 induced by MGCD0103 (Fig. 6C and D). The above results revealed that ROS was involved in the mitochondrial apoptosis but not in cell cycle arrest induced by MGCD0103.

\section{MGCD0103 results in autophagy correlated with cell death in liver cancer cells}

It has been reported that two HDAC inhibitors OSU-HDAC42 and SAHA induced autophagy in liver cancer cells and SAHA-induced autophagy led to cell death [22]. We found that MGCD0103, one of HDAC inhibitors, could also induce autophagy in HepG2 and Huh7 cells. Several autophagy-related proteins were tested by western blotting. MGCD0103 increased the expressions of RAGE, PI3KC3, Beclin 1, and LC3-II, and decreased that of p62 in a dose-dependent manner (Fig. 7A and B). The relative cell viability of HepG2 and Huh7 cells was significantly higher after exposure to 3-MA (a PI3KC3 inhibitor blocking autophagosome formation) and MGCD0103 than after treatment with MGCD0103 alone (Fig. 7C).

Addition of 3-MA (5 mM) increased the IC50 values of MGCD0103 in HepG2 cells for different lengths of time from $6.497 \pm 0.431$ to $14.497 \pm 0.732$ (24 h), from $1.427 \pm 0.206$ to $5.120 \pm 0.700$ ( $48 \mathrm{~h}$ ), and from $0.453 \pm 0.055$ to $2.037 \pm 0.111 \mu \mathrm{M}(72 \mathrm{~h})$, respectively (Fig. 7C). Consistent with the effect of 3-MA on HepG2 cells, 3-MA also increased the IC50 values of 
MGCD0103 in Huh7 cells from $4.567 \pm 0.496$ to 10.490 $\pm 0.520(24 \mathrm{~h}$ ), from $0.920 \pm 0.096$ to $3.887 \pm 0.669$ (48 h), and from $0.277 \pm 0.061$ to $1.470 \pm 0.142 \mu \mathrm{M}(72 \mathrm{~h})$, respectively (Fig. 7C). Apoptosis analysis showed that addition of 3-MA decreased the apoptotic rate from $28.47 \pm 2.85 \%$ caused by MGCD0103 to $18.08 \pm 1.88 \%$ in HepG2 cells and from $33.29 \pm 2.93$ to $19.53 \pm 1.55 \%$ in Huh7 cells (Fig. 7D). Western blotting revealed that 3-MA reduced the cleavage of PARP, accumulation of LC3-II induced by MGCD0103, and degration of p62 induced by autophagy (Fig. 7E and F). We then investigated the effect of Z-VAD-FMK and NAC on autophagy. Western blotting showed that whereas NAC attenuated accumulation of MGCD0103induced, autophagy-related proteins, Z-VAD-FMK exposure increased MGCD0103-induced, autophagyrelated proteins (Fig. 7G and $\mathrm{H}$ ).

\section{MGCD0103 inhibits tumor growth in animal model}

To further investigate the effect of MGCD0103 on liver cancer cell growth in vivo, HepG2 cells were subcutaneously injected into nude mice. MGCD0103 treatment was begun on the 9th day, and it was p.o.-administered daily at 50 or $100 \mathrm{mg} / \mathrm{kg}$ for 15 days. The results showed that MGCD0103 significantly suppressed the tumor volume (Fig. 8A and B). The tumor weight of MGCD0103-treated mice was much lighter than that of the control group (Fig. 8C). $\mathrm{Ki}-67$ and cleaved caspase- 3 staining of tumor sections was performed to evaluate tumor proliferation and apoptosis in vivo. MGCD0103 significantly decreased the percentage of $\mathrm{Ki}-67$ positive cells, and significantly increased the percentage of cleaved caspase-3 positive cells (Fig. 8D).

Afterwards we evaluated the toxicity after MGCD0103 treatment. We found that MGCD0103 exerted no obvious effect on body weight of mice and biochemical functions of liver and kidney (Fig. 8E and F).

Organs including heart, liver, spleen, lung, and kidney were stained with H\&E. Histological analysis showed no significant histological changes after MGCD0103 treatment (Fig. 8G).

\section{Discussion}

Several HDAC inhibitors, including those approved by FDA, have shown anticancer activity in liver cancer; a few relevant clinical trials are ongoing $[23,24]$. Although MGCD0103 is a HDAC inhibitor, its effect on liver cancer remains unknown. Zhou and his colleagues carried out pharmacokinetic studies of orally administered MGCD0103 in mice, rats, and dogs [25]. In their studies, they found that this compound had good oral bioavailability and was quickly absorbed across all species tested [25]. What's more, MGCD0103 showed significant anticancer activity in several tumor xenograft models in a dose-dependent manner and a more potent antiproliferative effect than MS-275 and SAHA in a number of human cancer cell lines [10]. In a phase 1 study in patients with hematologic malignancies, pharmacokinetic analyses demonstrated absorption of MGCD0103 within 1 hour after oral administration and a long elimination half-life in plasma of 9 plus or minus 2 hours [26]. Another phase 1 study of MGCD0103 in patients with advanced solid tumors found that its elimination terminal half-life ranged from 6.7 to 12.2 hours and no accumulation after repeated dosing was observed [11]. MGCD0103 undergoes hepatic metabolism and is eliminated via biliary and fecal routes [27].

In the present study, we found that MGCD0103, an isotype-specific HDAC inhibitor, had anticancer activity in liver cancer cell lines. This compound augmented the acetylated level of histone $\mathrm{H} 3$ and histone $\mathrm{H} 4$ by using western blotting. Moreover, we demonstrated that MGCD0103 significantly inhibited tumor growth in vivo and in vitro. This agent caused G2/M phase cell cycle arrest, apoptosis, and autophagy by affecting a series of related proteins (Fig. 9). MGCD0103 did not lead to organ-related and systemic toxicities in mice.

The hallmark of G2/M phase arrest is the deactivation of cdc25C and cdc2/cyclin B1 complex [28]. Cdc25C can dephosphorylate cdc2 and thus activate cdc2/cyclin B1 complex which promotes the G2/M transition. Phosphorylation of cdc25C and cdc2 reduces their activity and causes G2/M phase arrest. Furthermore, p21 and p27 can inhibit cdc2 activity to affect cell cycle $[29,30]$. In this study, cell cycle ananlysis showed that MGCD0103 resulted in G2/M arrest in a dose-dependent manner in HepG2 and Huh7 cells. Western blotting found that p21, p27, p-cdc25C, and p-cdc2 were upregulated and cdc25C, cdc2, and cyclin B1 were downregulated after treatment with MGCD0103. Changes in cell cycle-related proteins may induce G2/M phase arrest in MGCD0103-treated cells.

Apoptosis is a process of programmed cell death. Some factors like caspases induce apoptosis, while some members of the Bcl-2 family suppress apoptosis. Mitochondria apoptosis pathway is the major pathway involving in apoptosis. Mitochondria can produce ROS and release Cyto-C which activates its downstream caspases.

It has been reported that MGCD0103 exerts its anticancer activity by causing apoptosis in a variety of cancer cells [10, 31]. In our study, we found that MGCD0103 triggered apoptosis in a dose-dependent 
manner in HepG2 and Huh7 cells. Western blotting showed that proapoptotic proteins like Bim and Bax were upregulated and antiapoptotic proteins like Bcl-2 and Bcl-xL were downregulated after treatment with MGCD0103. What's more, expression of Cyto-C in cytosol was elevated in MGCD0103-treated cells. Following the treatment with MGCD0103, a significant increase of cleavage of caspase-9, -3, -7, and PARP was also observed. The caspase inhibitor Z-VAD-FMK and the ROS inhibitor NAC partially attenuated MGCD0103-induced apoptosis. The above results suggested that mitochondria participated in the apoptotic process induced by MGCD0103.

Apart from apoptosis, autophagy is a major form of programmed cell death. Previous studies have found that, during the process of autophagy, the expression of the receptor for advanced glycation end products (RAGE), Beclin 1, PI3KC3, and LC3-II is increased $[22,32]$. Meanwhile, p62 can be degraded by autophagy [33]. In the present study, we demonstrated that MGCD0103 elicited autophagy in HepG2 and Huh7 cells. Western blotting revealed the overexpression of RAGE, PI3KC3, Beclin 1, and LC3-II and the decreased expression of p62. 3-MA, the PI3KC3 inhibitor, partially reversed autophagy and autophagic cell death induced by MGCD0103.

Further study revealed that NAC reversed MGCD0103-induced autophagy, which indicated a link between ROS and autophagy. The complex interplay between autophagy and apoptosis depends on various cellular settings. Flow cytometric analysis and western blotting demonstrated that autophagy induced by MGCD0103 contributed to apoptosis in liver cancer cells; and that Z-VAD-FMK further increased MGCD0103-induced autophagy even more, indicating that, when apoptosis was inhibited, autophagic cell death was prominent in liver cancer cells. Consequently, in our study, both apoptosis and autophagy induced by MGCD0103 cooperatively led to liver cancer cell death, with autophagy serving as a back-up mechanism when apoptosis was defective. The mechanisms that underlie the crosstalk between apoptosis and autophagy warrant further investigation.

Although most HDAC inhibitors approved by FDA are nonselective, MGCD0103, an isotypeselective HDAC inhibitor, targets HDAC1, HDAC2, HDAC3 and HDAC11. Our results indicate that anticancer efficacy by a HDAC inhibitor did not require that it inhibit all HDAC isotypes. Inhibition of certain HDAC isoforms by MGCD0103 was enough for antitumor activity in this study, and suggests that clinical use of less-comprehensive HDAC inhibitors for liver cancer and other cancers might offer efficacy with less toxicity.
$\mathbf{A}$

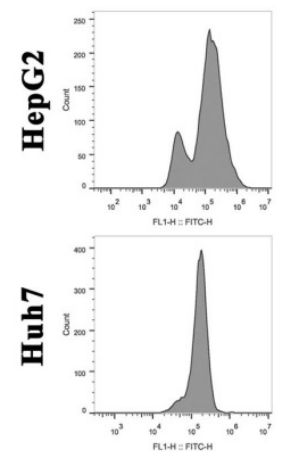

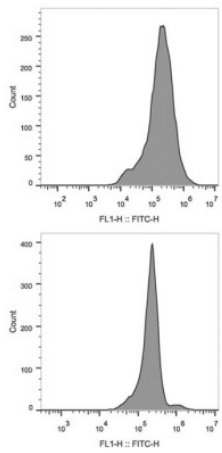

B
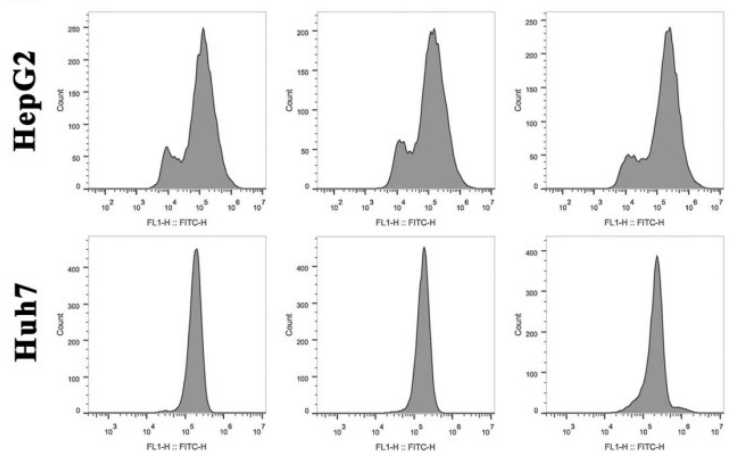
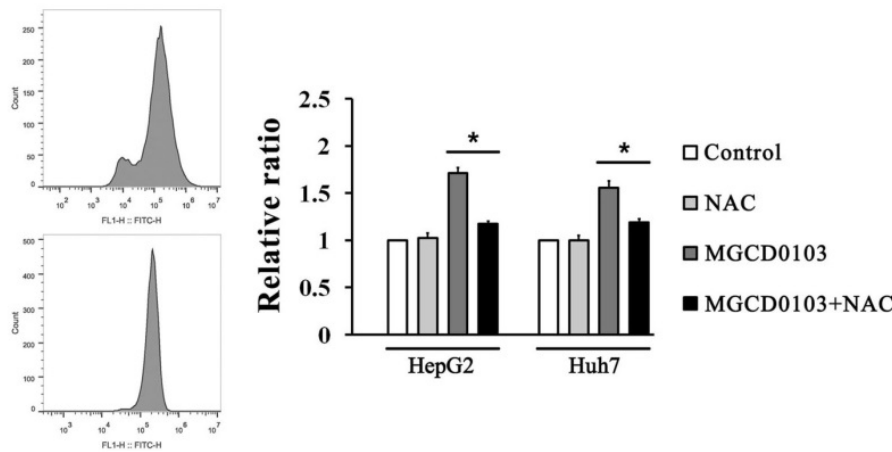

Figure 5. ROS inhibitor NAC partially decreased MGCD0103-induced ROS levels in liver cancer cells. First, HepG2 and Huh7 cells were treated with increasing concentrations $(0,1$, and $5 \mu \mathrm{M})$ of MGCD0103 for $48 \mathrm{~h}$. Then cells were pretreated with $5 \mathrm{mM} \mathrm{NAC}$ for $1 \mathrm{~h}$ and then treated with $5 \mu \mathrm{M}$ MGCD0103 for the next $48 \mathrm{~h}$. (A, B) ROS levels were evaluated by flow cytometry. 
A
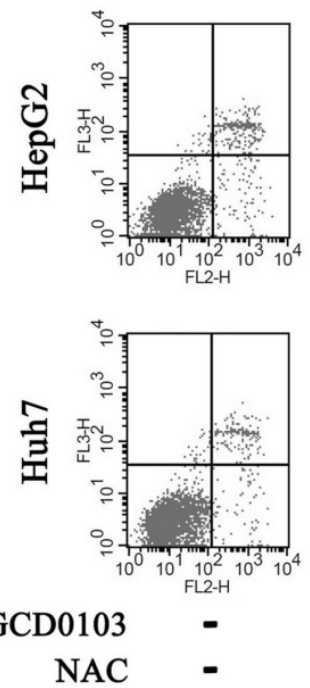

B
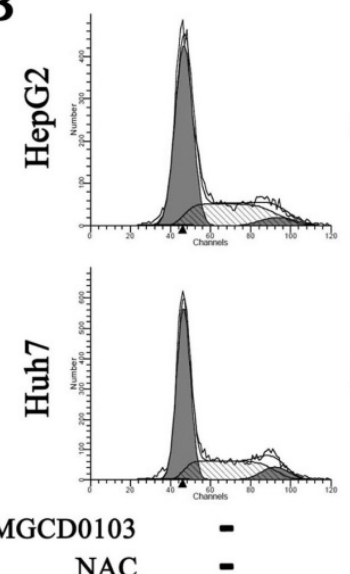

C

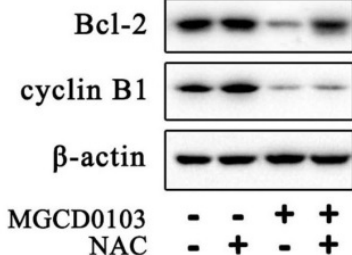

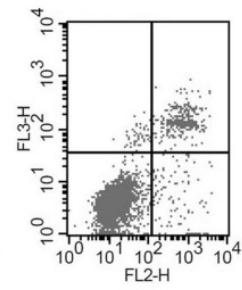
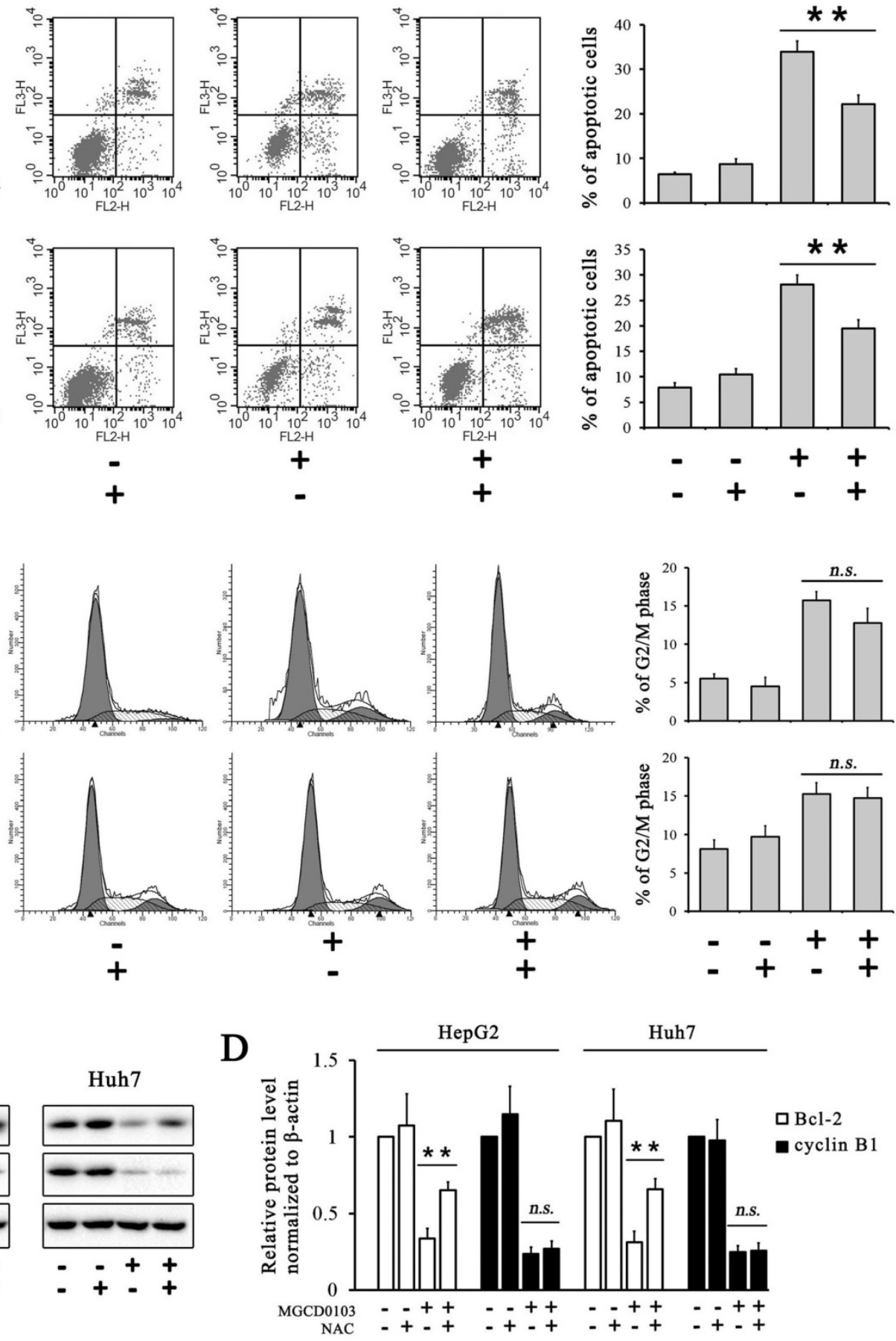

Figure 6. ROS inhibitor NAC partially inhibited apoptosis induced by MGCD0103 in liver cancer cells. Cells were pretreated with $5 \mathrm{mM}$ NAC for $1 \mathrm{~h}$ and then treated with $5 \mu \mathrm{M}$ MGCD0103 for the next $48 \mathrm{~h}$. (A) Apoptosis was evaluated by flow cytometry. (B) Cell cycle distribution was evaluated by flow cytometry. (C, D) Western blotting analysis of $\mathrm{Bcl}-2$ and cyclin $\mathrm{B} 1$ after MGCD0103 and/or NAC treatment. $* P<0.05 ; * * P<0.01$

In addition to efficacy, safety is an increasing concern for HDAC inhibitors. MGCD0103 is a rationally designed and orally bioavailable HDAC inhibitor [25]. Expressions of fewer genes are induced by MGCD0103, a benzamide HDAC inhibitor, than are induced by nonselective hydroxamate HDAC inhibitors, yet the efficacy of MGCD0103 in preclinical tumor models is maintained or even elevated [10]. Possibly because of its slow-tight kinetic properties, the off-binding rate of MGCD0103 is very low, so that its inhibitory effect is prolonged after its removal. Therefore, patients can receive lower doses and less frequent dosing of MGCD0103 while its pharmacodynamic effect is still maintained [34].

In brief, MGCD0103 may have advantages over broad-spectrum HDAC inhibitors due to longer half-life, good oral bioavailability and greater therapeutic index. 

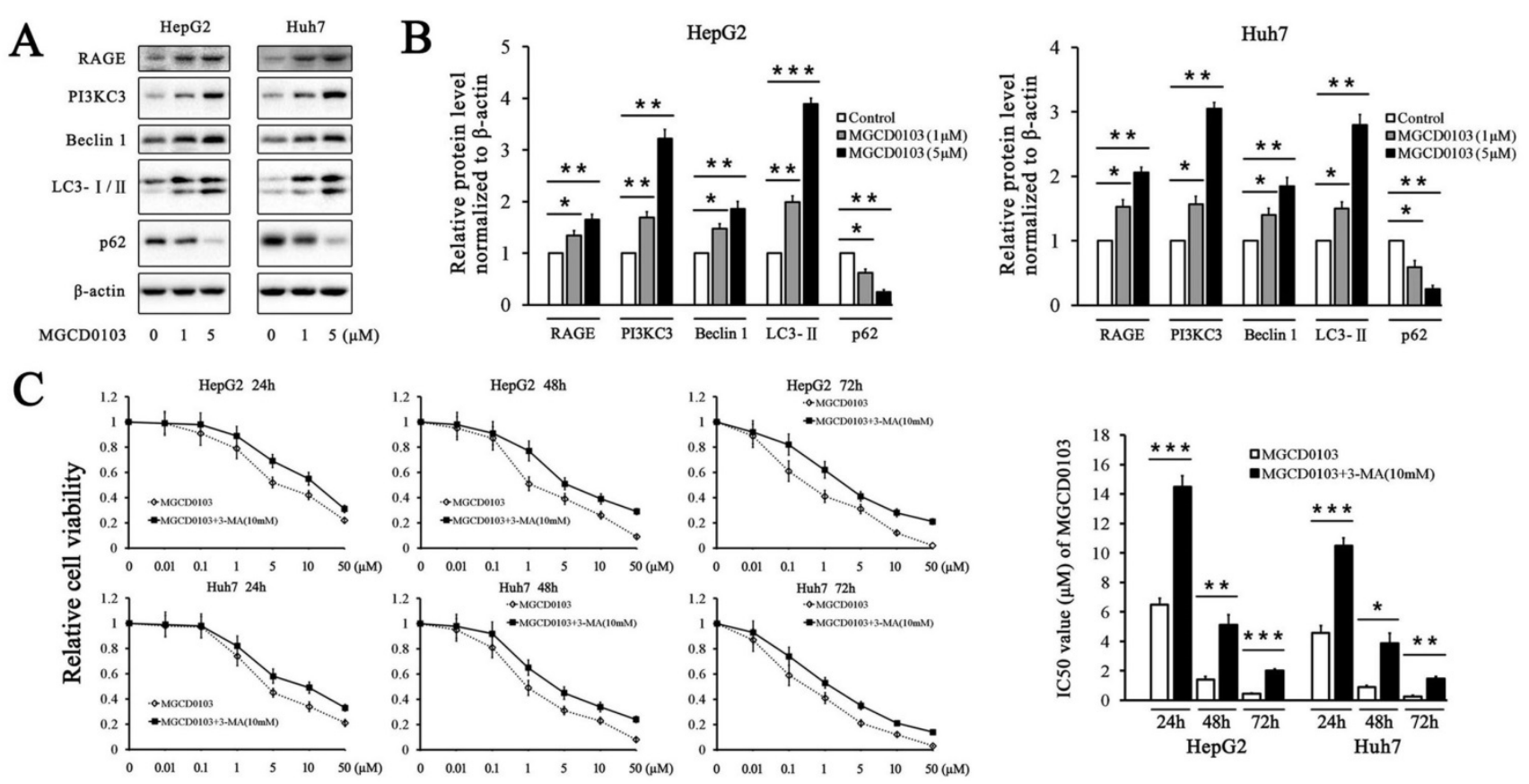

D

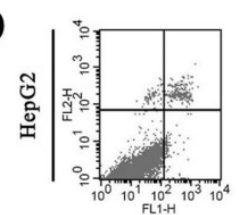

MGCD0103

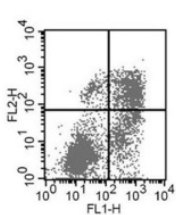

$+$

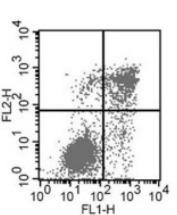

$+$

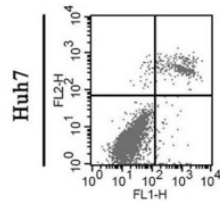

-

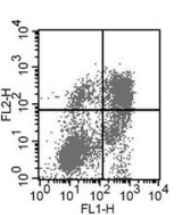

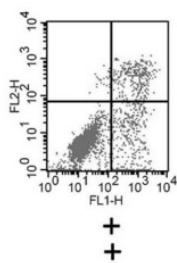

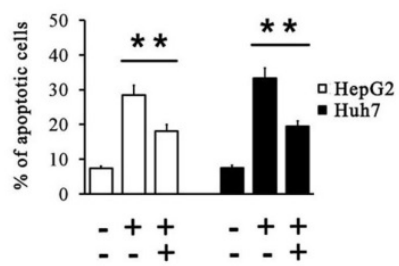

HepG2

$\mathrm{E}$

Huh7

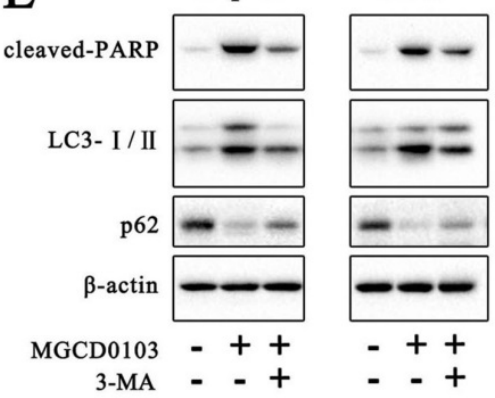

$\mathbf{F}$
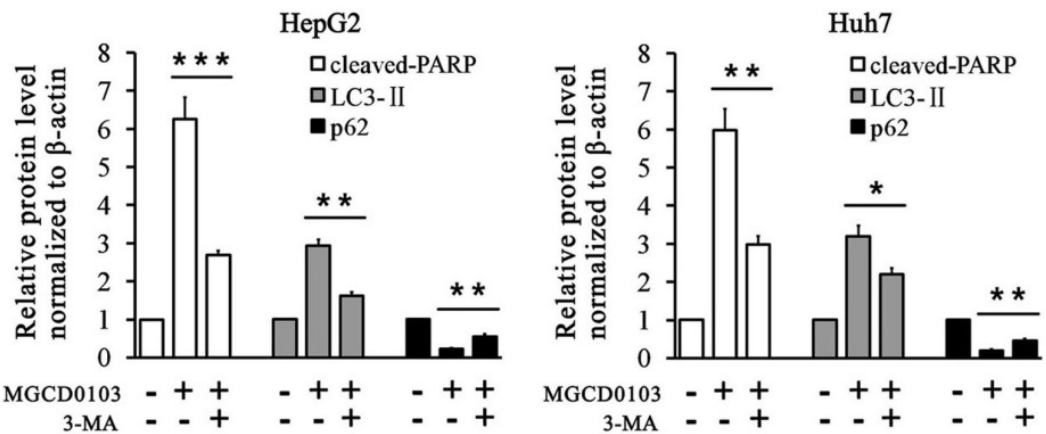

Huh7

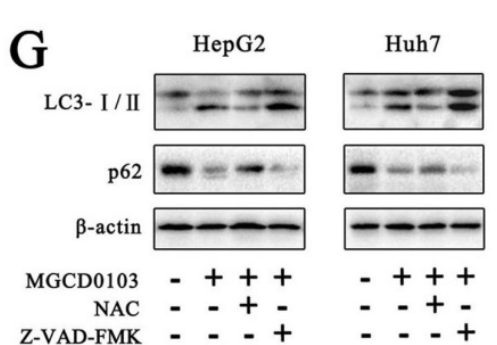

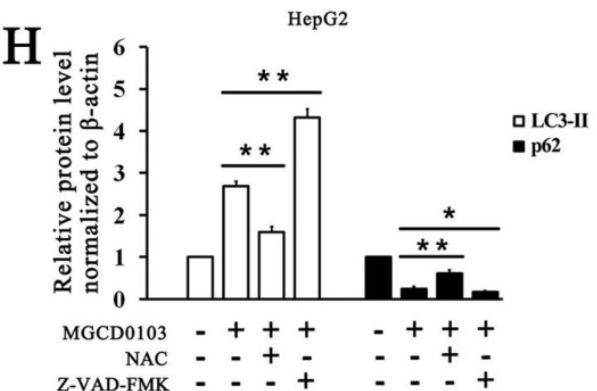

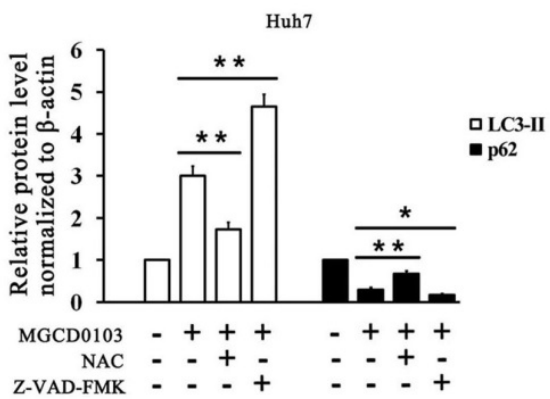

Figure 7. MGCD0103 causes autophagy and autophagic cell death in liver cancer cells. (A, B) HepG2 and HUH7 cells were treated with MGCD0103 (1 $\mu$ M and $5 \mu \mathrm{M}$ ) for $48 \mathrm{~h}$. Several autophagy-related proteins including RAGE, PI3KC3, Beclin 1, LC3-I/II, and p62 were then analyzed using western blotting. (C) HepG2 and Huh7 cells were treated with increasing concentrations $(0.01,0.1,1,5,10$, and $50 \mu \mathrm{M})$ of MGCD0103 alone or in combination with $5 \mathrm{mM} 3-\mathrm{MA}$ for $24 \mathrm{~h}, 48 \mathrm{~h}$, or $72 \mathrm{~h}$. Cell viability was assessed using the CCK-8 assay. The IC50 values of MGCD0103 were calculated and compared. (D) HepG2 and Huh7 cells were treated with $5 \mu$ M MGCD0103 alone or in combination with $5 \mathrm{mM}$ 3-MA for $48 \mathrm{~h}$. Apoptosis was evaluated by flow cytometry. (E, F) HepG2 and Huh7 cells were treated with 5 HM MGCD0103 alone or in combination with $5 \mathrm{mM}$ 3-MA for $48 \mathrm{~h}$. The protein level of cleaved-PARP, LC3-I/II, and p62 was tested by western blotting. (G, H) HepG2 and Huh7 cells were treated with MGCD0103 $(5 \mu M)$ in the presence or absence of NAC $(5 \mathrm{mM})$ and Z-VAD-FMK $(20 \mu \mathrm{M})$ for $48 \mathrm{~h}$. The protein level of LC3-I/ll and $p 62$ was tested by western blotting. $* P<0.05 ; * * P<0.01 ; * * * P<0.001$ 


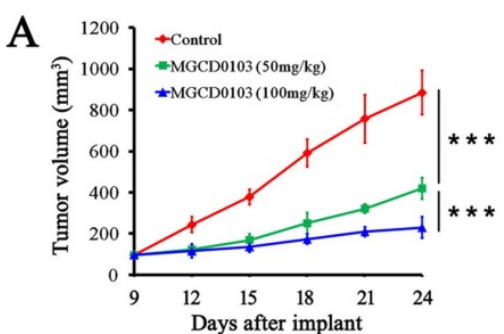

B

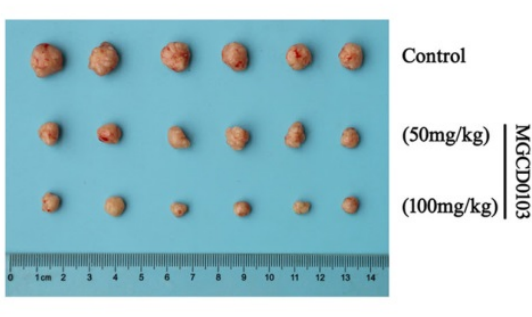

C

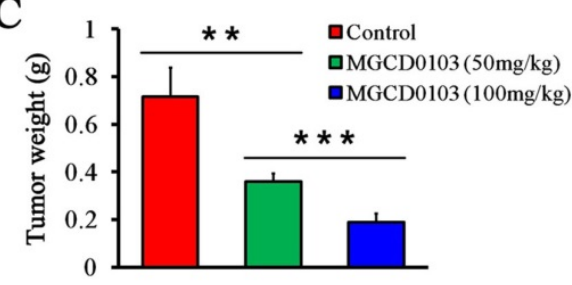

D
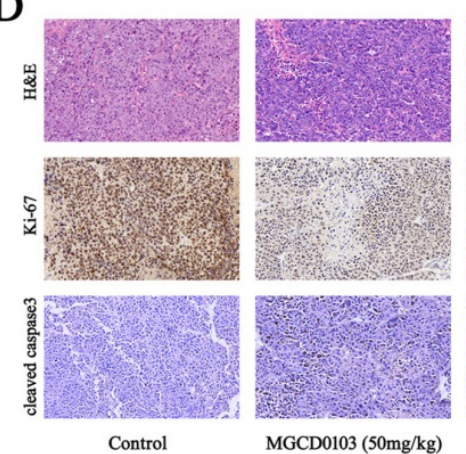

$\operatorname{MGCD} 0103(50 \mathrm{mg} / \mathrm{kg})$
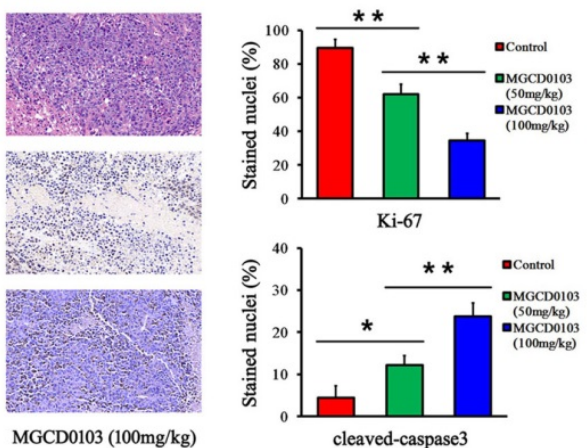

E

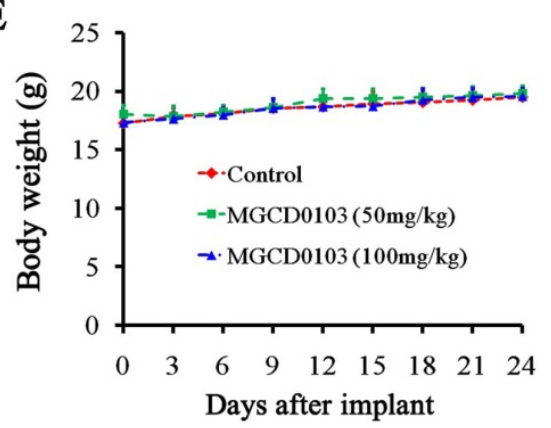

$\mathbf{F}$

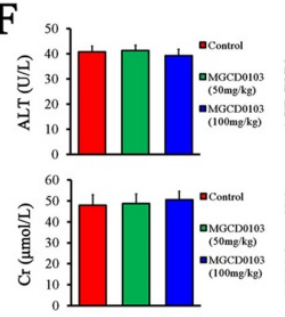

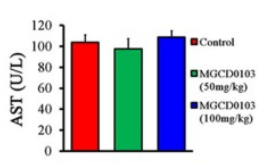

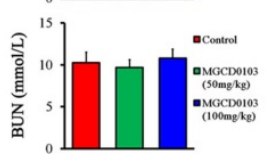

G

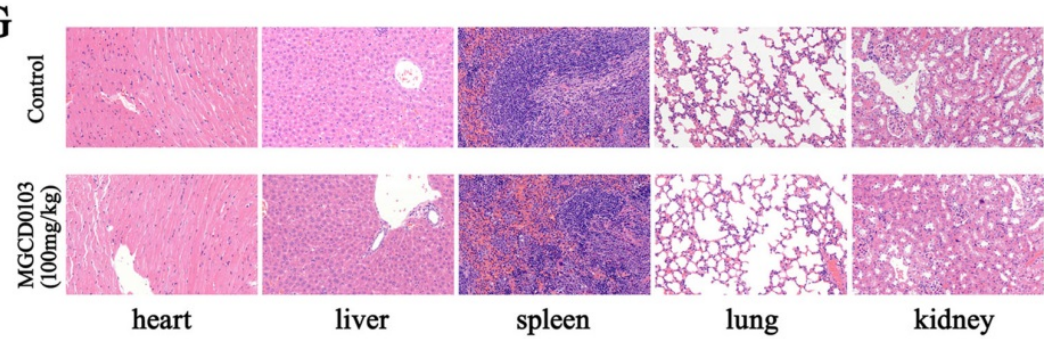

Figure 8. MGCD0103 inhibited tumorigenicity in vivo. (A) The tumor growth curve of HepG2 cells. MGCD0103 treatment was initiated 9 days after tumor implantation. Tumor volume was calculated every 3 days. Mice were randomized into three groups $(n=6)$ and MGCD0103 was dosed per os (p.o.) daily at 50 or 100 mg/kg. (B) Representative images of the subcutaneous tumors in the three groups. (C) Tumor weights in the different groups were measured and compared. (D) Representative images of H\&E, Ki-67, and cleaved caspase-3 staining of tumor sections. The percentage of $\mathrm{Ki}-67$ and cleaved caspase-3 positive cells was calculated and compared in the different groups. (E) The body weight curve of mice in the three groups. Body weight was measured every 3 days. (F) The levels of AST, ALT, Cr, and BUN of mice blood were detected by biochemistry analyzer. (G) Representative images of H\&E staining of heart, liver, spleen, lung, and kidney. The histologic structures were observed and compared microscopically. $* P<0.05$; $* * p<0.01 ; * * * p<0.001$

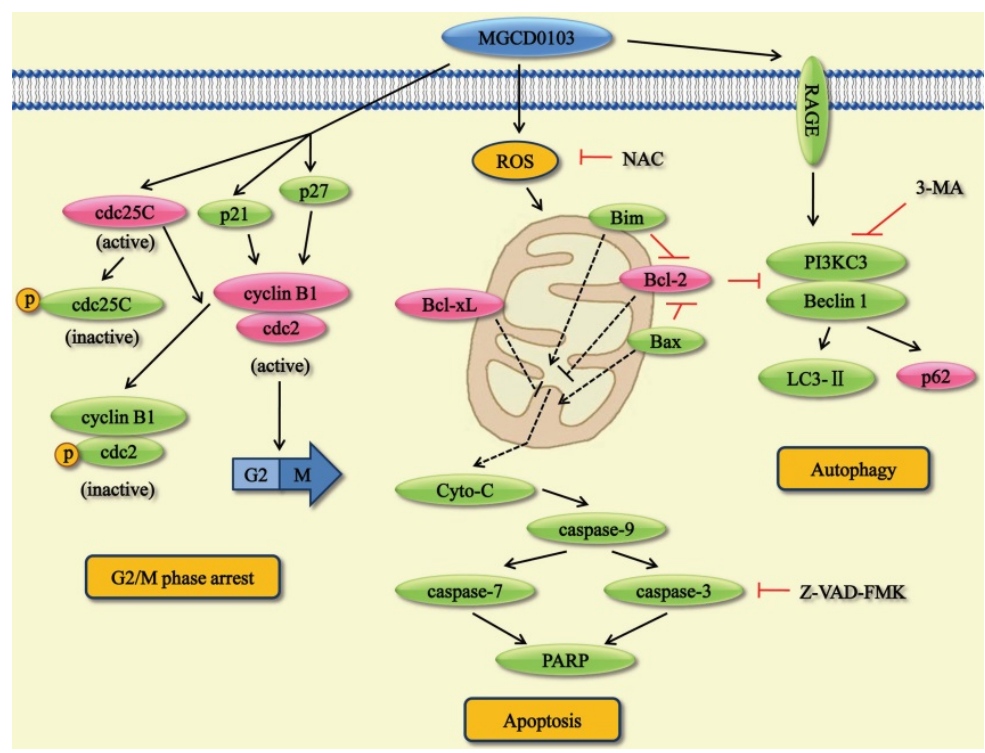

Figure 9. Overview of the pathways for MGCD0103-induced cell cycle arrest, apoptosis, and autophagy in liver cancer cells.

\section{Conclusions}

In summary, the present study investigated the molecular mechanisms underlying the anticancer effect of MGCD0103 on liver cancer cells. MGCD0103 could cause G2/M phase arrest via cdc25C/ cdc2/cyclinB pathway, trigger apoptosis via mitochondrial pathway, and induce autophagy via RAGE/PI3KC3/Beclin 1 pathway. Furthermore, MGCD0103 could significantly inhibit the growth of liver cancer in vitro and in vivo. Consequently, MGCD0103 may be a novel and efficacious agent against liver cancer.

\section{Abbreviations}

HDAC: histone deacetylase; HAT: histone acetyl transferase; 3-MA: 3-Methyl- 
adenine; NAC: N-Acetyl Cysteine; CCK-8: Cell Counting Kit-8; IC50: half maximal inhibitory concentration; RAGE: receptor for advanced glycation end products.

\section{Acknowledgements}

This study was supported by the Natural Science Foundation of Hubei Province of China (Grant No. 2019CFB707) and the Fundamental Research Funds for the Central Universities of Ministry of Education of China (Grant No. 2042017kf0094).

\section{Competing Interests}

The authors have declared that no competing interest exists.

\section{References}

1. Torre LA, Bray F, Siegel RL, Ferlay J, Lortet-Tieulent J, Jemal A. Global cancer statistics, 2012. CA Cancer J Clin. 2015;65:87-108.

2. Yang JD, Roberts LR. Hepatocellular carcinoma: A global view. Nat Rev Gastroenterol Hepatol. 2010;7:448-58.

3. Forner A, Llovet JM, Bruix J. Hepatocellular carcinoma. Lancet. 2012;379:1245-55.

4. Chen PJ, Furuse J, Han KH, Hsu C, Lim HY, Moon H, et al. Issues and controversies of hepatocellular carcinoma-targeted therapy clinical trials in Asia: experts' opinion. Liver Int. 2010;30:1427-38.

5. Poon D, Anderson BO, Chen LT, Tanaka K, Lau WY, Van Cutsem E, et al. Management of hepatocellular carcinoma in Asia: consensus statement from the Asian Oncology Summit 2009. Lancet Oncol. 2009;10:1111-8.

6. Hanahan D, Weinberg RA. Hallmarks of cancer: the next generation. Cell. 2011;144:646-74.

7. Jones PA, Baylin SB. The epigenomics of cancer. Cell. 2007;128:683-92.

8. Lakshmaiah KC, Jacob LA, Aparna S, Lokanatha D, Saldanha SC. Epigenetic therapy of cancer with histone deacetylase inhibitors. J Cancer Res Ther. 2014;10:469-78.

9. Li Y, Seto E. HDACs and HDAC Inhibitors in Cancer Development and Therapy. Cold Spring Harb Perspect Med. 2016:6:a026831.

10. Fournel M, Bonfils C, Hou Y, Yan PT, Trachy-Bourget MC, Kalita A, et al. MGCD0103, a novel isotype-selective histone deacetylase inhibitor, has broad spectrum antitumor activity in vitro and in vivo. Mol Cancer Ther. 2008;7:759-68.

11. Siu LL, Pili R, Duran I, Messersmith WA, Chen EX, Sullivan R, et al. Phase I study of MGCD0103 given as a three-times-per-week oral dose in patients with advanced solid tumors. J Clin Oncol. 2008;26:1940-7.

12. Younes A, Oki Y, Bociek RG, Kuruvilla J, Fanale M, Neelapu S, et al. Mocetinostat for relapsed classical Hodgkin's lymphoma: an open-label, single-arm, phase 2 trial. Lancet Oncol. 2011;12:1222-8.

13. Batlevi CL, Crump M, Andreadis C, Rizzieri D, Assouline SE, Fox S, et al. A phase 2 study of mocetinostat, a histone deacetylase inhibitor, in relapsed or refractory lymphoma. Br J Haematol. 2017;178:434-41.

14. El-Khoury V, Pierson S, Szwarcbart E, Brons NH, Roland O, Cherrier-De Wilde S, et al. Disruption of autophagy by the histone deacetylase inhibitor MGCD0103 and its therapeutic implication in B-cell chronic lymphocytic leukemia. Leukemia. 2014:28:1636-46.

15. Wei Y, Kadia T, Tong W, Zhang M, Jia Y, Yang H, et al. The combination of a histone deacetylase inhibitor with the Bcl-2 homology domain-3 mimetic GX15-070 has synergistic antileukemia activity by activating both apoptosis and autophagy. Clin Cancer Res. 2010;16:3923-32.

16. Ding ZY, Jin GN, Wang W, Chen WX, Wu YH, Ai X, et al. Reduced expression of transcriptional intermediary factor 1 gamma promotes metastasis and indicates poor prognosis of hepatocellular carcinoma. Hepatology. 2014;60:1620-36

17. Chen L, Zhang W, Zhou QD, Yang HQ, Liang HF, Zhang BX, et al. HSCs play a distinct role in different phases of oval cell-mediated liver regeneration. Cell Biochem Funct. 2012;30:588-96.

18. Wei S, Xiong M, Zhan DQ, Liang BY, Wang YY, Gutmann DH, et al. Ku80 functions as a tumor suppressor in hepatocellular carcinoma by inducing S-phase arrest through a p53-dependent pathway. Carcinogenesis. 2012;33:538-47.

19. Li GZ, Liang HF, Liao B, Zhang L, Ni YA, Zhou HH, et al. PX-12 inhibits the growth of hepatocelluar carcinoma by inducing S-phase arrest, ROS-dependent apoptosis and enhances 5-FU cytotoxicity. Am J Transl Res. 2015;7:1528-40.
20. Zhang B, Halder SK, Kashikar ND, Cho YJ, Datta A, Gorden DL, et al. Antimetastatic role of Smad4 signaling in colorectal cancer. Gastroenterology. 2010;138:969-80 e1-3.

21. Fleury C, Mignotte B, Vayssiere JL. Mitochondrial reactive oxygen species in cell death signaling. Biochimie. 2002;84:131-41.

22. Liu YL, Yang PM, Shun CT, Wu MS, Weng JR, Chen CC. Autophagy potentiates the anti-cancer effects of the histone deacetylase inhibitors in hepatocellular carcinoma. Autophagy. 2010;6:1057-65.

23. Liu KY, Wang LT, Hsu SH. Modification of Epigenetic Histone Acetylation in Hepatocellular Carcinoma. Cancers (Basel). 2018;10:8

24. Tsilimigras DI, Ntanasis-Stathopoulos I, Moris D, Spartalis E, Pawlik TM. Histone deacetylase inhibitors in hepatocellular carcinoma: A therapeutic perspective. Surg Oncol. 2018;27:611-8.

25. Zhou N, Moradei O, Raeppel S, Leit S, Frechette S, Gaudette F, et al. Discovery of N-(2-aminophenyl)-4-[(4-pyridin-3-ylpyrimidin-2 -ylamino)methyl]benzamide (MGCD0103), an orally active histone deacetylase inhibitor. J Med Chem. 2008;51:4072-5.

26. Garcia-Manero G, Assouline S, Cortes J, Estrov Z, Kantariian H, Yang H, et al. Phase 1 study of the oral isotype specific histone deacetylase inhibitor MGCD0103 in leukemia. Blood. 2008;112:981-9.

27. Lane AA, Chabner BA. Histone deacetylase inhibitors in cancer therapy. J Clin Oncol. 2009;27:5459-68.

28. Perdiguero E, Nebreda AR. Regulation of Cdc25C activity during the meiotic G2/M transition. Cell Cycle. 2004;3:733-7.

29. Bunz F, Dutriaux A, Lengauer C, Waldman T, Zhou S, Brown JP, et al. Requirement for p53 and p21 to sustain G2 arrest after DNA damage. Science. 1998;282:1497-501.

30. Hsu JD, Kao SH, Ou TT, Chen YJ, Li YJ, Wang CJ. Gallic acid induces G2/M phase arrest of breast cancer cell MCF-7 through stabilization of p27(Kip1) attributed to disruption of p27(Kip1)/Skp2 complex. J Agric Food Chem. 2011;59:1996-2003.

31. El-Khoury V, Moussay E, Janji B, Palissot V, Aouali N, Brons NH, et al. The histone deacetylase inhibitor MGCD0103 induces apoptosis in B-cell chronic lymphocytic leukemia cells through a mitochondria-mediated caspase activation cascade. Mol Cancer Ther. 2010:9:1349-60.

32. Kang R, Tang D, Schapiro NE, Livesey KM, Farkas A, Loughran P, et al. The receptor for advanced glycation end products (RAGE) sustains autophagy and limits apoptosis, promoting pancreatic tumor cell survival. Cell Death Differ. 2010;17:666-76.

33. Bjorkoy G, Lamark T, Brech A, Outzen H, Perander M, Overvatn A, et al. p62/SQSTM1 forms protein aggregates degraded by autophagy and has a protective effect on huntingtin-induced cell death. J Cell Biol. 2005;171:603-14.

34. Bonfils C, Kalita A, Dubay M, Siu LL, Carducci MA, Reid G, et al. Evaluation of the pharmacodynamic effects of MGCD0103 from preclinical models to human using a novel HDAC enzyme assay. Clin Cancer Res. 2008;14:3441-9. 